\title{
KALATHOI IBERICI DA TAORMINA. AGGIORNAMENTO SULLA DIFFUSIONE DELLA CERAMICA IBERICA DIPINTA IN SICILIA
}

POR

\author{
FRANCESCO MUSCOLINO
}

\section{RÉSUMÉ}

La pubblicazione di alcuni frammenti di sombreros de copa da Taormina offre lo spunto per riconsiderare globalmente la presenza di analoghi manufatti in Sicilia.

\section{RESUMEN}

La publicación de algunos fragmentos de sombreros de copa hallados en Taormina presenta la oportunidad de considerar globalmente el fenómeno de su presencia en Sicilia.

PAROLE CHIAVE: Kalathoi iberici; Sicilia; Taormina

PALABRAS CLAVE: Kalathoi ibéricos; Sicilia; Taormina.

Alla cara memoria di Delia e Cristina Bolognari

I primi frammenti di kalathoi o sombreros de copa iberici identificati come tali in Sicilia provengono, forse non casualmente, ${ }^{1}$ dagli scavi condotti a Tindari nel 1952 da N. Lamboglia, autore, nel 1954, di un fondamentale articolo sulla ceramica iberica. ${ }^{2}$ I frammenti ${ }^{3}$ di Tindari, pubblicati da M.A. Mezquiriz, ${ }^{4}$

\footnotetext{
* Ringrazio cordialmente la Dott.ssa Maria Costanza Lentini per il permesso di pubblicare i frammenti di Taormina. I miei ringraziamenti vanno anche alla Prof.ssa Maria Paz García-Bellido per l'interesse mostrato, sin dall'inizio, per questo contributo. I disegni sono di Concetta Marano, che ringrazio per la sua amichevole collaborazione.

${ }^{1}$ «Non è ... un caso, credo, che (in Italia) la ceramica iberica sia stata in pratica rinvenuta laddove è stata cercata con attenzione; così, pertanto, dobbiamo ad esempio la maggioranza dei rinvenimenti conosciuti al Lamboglia» (Bencivenga Trillmich, 1984, 25, n. 20).

2 Lamboglia, 1954.

3 Sono, in totale, sei frammenti riferibili a quattro kalathoi: tre frammenti appartengono a tre diversi esemplari, e gli altri tre allo stesso kalathos (Mezquiriz, 1953, 158-159).

4 Mezquiriz, 1953; García y Bellido, 1954, 251; Lamboglia, 1954, 125; Nordström, 1973, 178; Montanya Maluquer, Puig Ochoa, 1979, 223; Santos Velasco, 1982-1983, 143; Bencivenga Trillmich, 1984, 28, n 19; Ead., 1985, 552, n 19 ; Ros Sala, 1989, 79; Bruni, Conde, 1991, 563, fig. 4; Conde Berdós, 1991, 154, fig. 14; Ead., 1992, 143, fig. 1 e 146, fig. 4.
}

dopo un accenno preliminare di A. García y Bellido, ${ }^{5}$ provengono dallo strato III, databile nella prima metà del I sec. a. C., di un sondaggio effettuato a ridosso della cortina interna delle mura di Tindari. ${ }^{6}$

Quattro frammenti (inv. 18874) di altrettanti kalathoi sono stati ritrovati nel taglio 10 della zona AR dell' Acropoli di Lipari, in scavi diretti da L. Bernabò Brea nel 1952. Tre di questi frammenti, pubblicati da M.A. Mezquiriz, ${ }^{7}$ dopo una notizia preliminare, ${ }^{8}$ sono stati ripubblicati in Meligunis Lipára IX.1, ${ }^{9}$ con l'aggiunta del quarto frammento, e con una datazione «fra il II e il I sec. a.C.» ${ }^{10}$ proposta per confronto, a causa della mancanza di dati stratigrafici accettabili. Altri quattro frammenti, appartenenti anch'essi, a quanto sembra, ad altrettanti kalathoi, provengono dallo scavo dell'aggere di Sesto Pompeo, nella città bassa, e sono datati, per l'associazione con ceramica Campana A e C, al II-I sec. a.C. ${ }^{11}$

5 García y Bellido, 1952, 45.

6 Mezquiriz, 1953, 159-160.

7 Mezquiriz Irujo, 1955. Le illustrazioni, che dovevano comparire in questo articolo, sono invece pubblicate in García y Bellido, 1957, 93. I frammenti editi da M.A. Mezquriz sono noti, oltre che a García y Bellido, 1957, 93-94, anche a Nordström, 1973, 178; Santos Velasco, 1982-1983, 143; Bencivenga Trillmich, 1984, 27, ${ }^{\circ} 18$; Ead., 1985, 552, n ${ }^{\circ}$ 18; Conde Berdós, 1992, 143, fig. 1.

8 Mezquiriz, 1953, 161, n. 10. La studiosa parla in realtà di ceramica iberica "con la representación de un pájaro", affermazione che non può riferirsi ai frammenti editi in Mezquiriz Irujo, 1955. È possibile che tale affermazione sia da attribuire alla mancata autopsia dei reperti successivamente editi. Un frammento inedito è citato anche da Lamboglia 1954, 125.

9 García y Bellido, 1957, 93, frammento a sinistra in alto = Meligunis Lipára IX.1, tav. 72.2, $\mathrm{n}^{\circ} 1$ o 2; García y Bellido, 1957, 93, frammento a sinistra in basso $=$ Meligunis Lipára IX.1, tav. 72.2. $\mathrm{n}^{\circ}$ 3; García y Bellido, 1957, 93, frammento a destra = Meligunis Lipára IX.1, tav. 72.2, n 4.

${ }^{10}$ Meligunis Lipára IX.1, 140, tav. 57.2.

${ }^{11}$ Meligunis Lipára IX.2, 162, tav. 206.3 a-d. Due frammenti provengono dal quadrato 4M/71, uno dal quadrato 4K-72, t. 6-8, e uno dallo scavo Cirotti XL-87 E, t. 3-5, 14-17. 

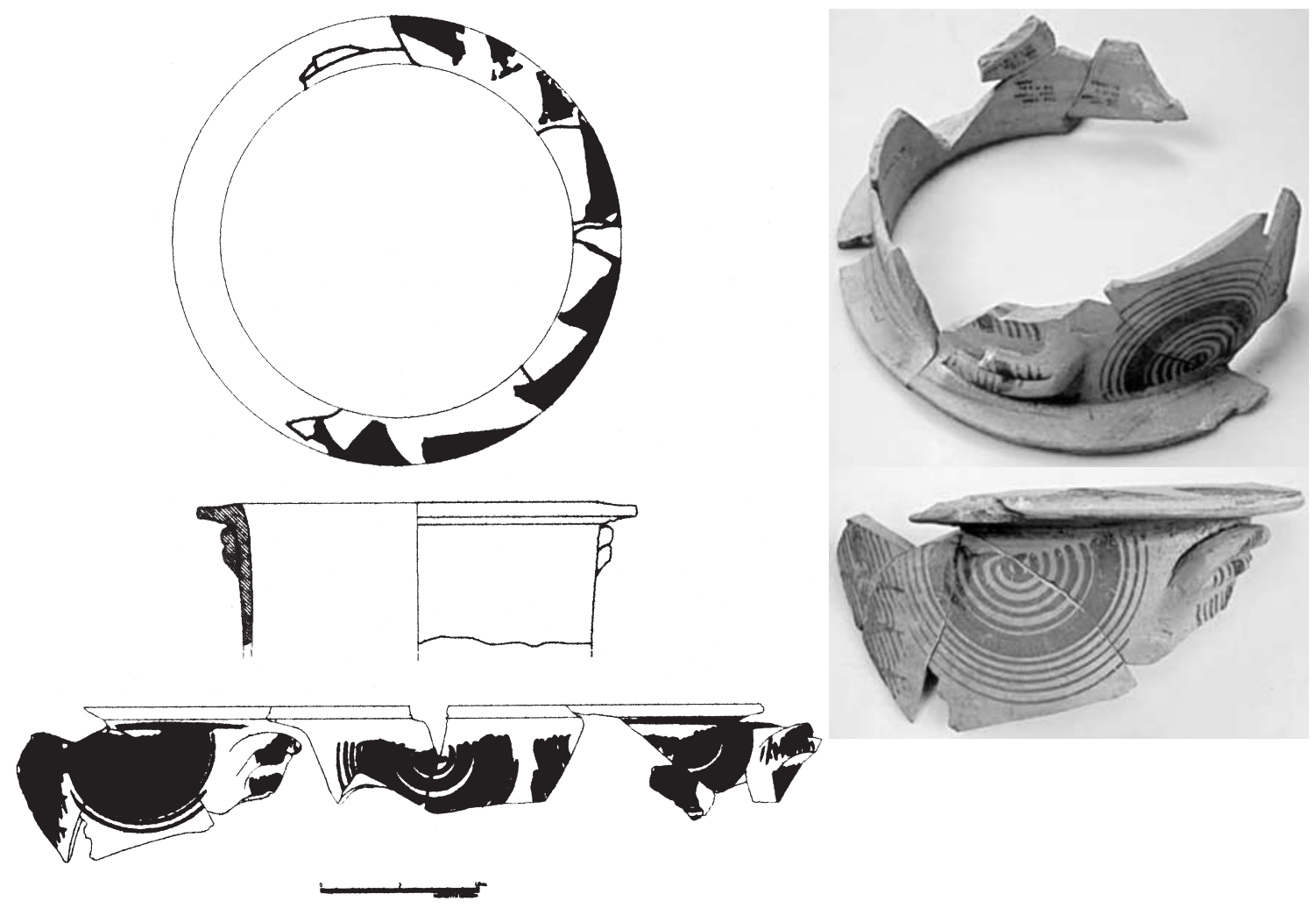

Figura 1. Ricostruzione del kalathos $\mathrm{n}^{\circ} 1$.

Altri frammenti di ceramica iberica, appartenenti a un kalathos o forse due, ${ }^{12}$ sono stati rinvenuti ad Heraclea Minoa negli scavi condotti tra 1955 e 1957, «nel terreno accumulatosi sulla fronte del muro di fortificazione, tratto $\mathrm{C}$ », e sono stati pubblicati da $\mathrm{E}$. De Miro, con datazione ad «età ellenistica». ${ }^{13}$

Un numero imprecisato ma cospicuo di frammenti di kalathoi iberici proviene da Lilibeo, ma di essi si ha soltanto una sintetica menzione pubblicata da C.A. Di Stefano ${ }^{14}$. Un gruppo di frammenti è stato rinvenuto in uno scavo di emergenza condotto nel 1974 nella necropoli, un altro gruppo proviene dall'abitato, da una

12 Tre frammenti combacianti (inv. 689) e un frammento (inv. 689 b) non combaciante ma appartenente forse allo stesso esemplare.

${ }^{13}$ De Miro, 1958, 272-273, fig. 39. I frammenti di Heraclea sono noti a Bencivenga Trillmich, 1984, 28, n 20; Ead., 1985, 552, n 20; Bruni, Conde, 1991, 563, fig. 4; Conde Berdós, 1991, 154, fig. 14; Ead., 1992, 143, fig. 1 e 146, fig. 4.

${ }^{14}$ Di Stefano, 1976, 29-30, fig. 4. La ceramica iberica di Lilibeo non risulta citata nella bibliografia consultata per la redazione di questo testo. cisterna, indagata nei primi anni Settanta, che ha restituito anche ceramica a vernice nera. Il primo gruppo di reperti lilibetani sembrerebbe l'unico proveniente, in Sicilia, da un'area di necropoli.

A questi esemplari è ora possibile aggiungere $\mathrm{i}$ frammenti di tre o quattro kalathoi rinvenuti a Taormina in una cisterna indagata nel maggio 2001 nel giardino dell'Hôtel Timeo, a poca distanza dal Teatro antico. Il materiale rinvenuto all'interno della cisterna sembra databile, almeno in prevalenza, al II sec. a.C.

Tutti i frammenti di kalathoi iberici provengono dal livello più basso della cisterna, compreso tra m. -3.00 dall'imboccatura e il fondo (- m. 4.00), tranne uno dei nove frammenti combacianti del kalathos $\mathrm{n}^{\circ} 1$, che proviene dal livello tra m. -2.10 e -2.40 dall'imboccatura. L'argilla, rosata ${ }^{15}$ in superficie, è ben depurata, compatta, con linee di frattura piuttosto nette. La vernice, abbastanza densa e stesa piuttosto uniformemente, è di colore rosso. ${ }^{16}$

\footnotetext{
${ }^{15}$ Confrontabile con M. 7.5YR 7/3-7/4.

${ }^{16}$ Confrontabile con M. 10R 4/6.
} 


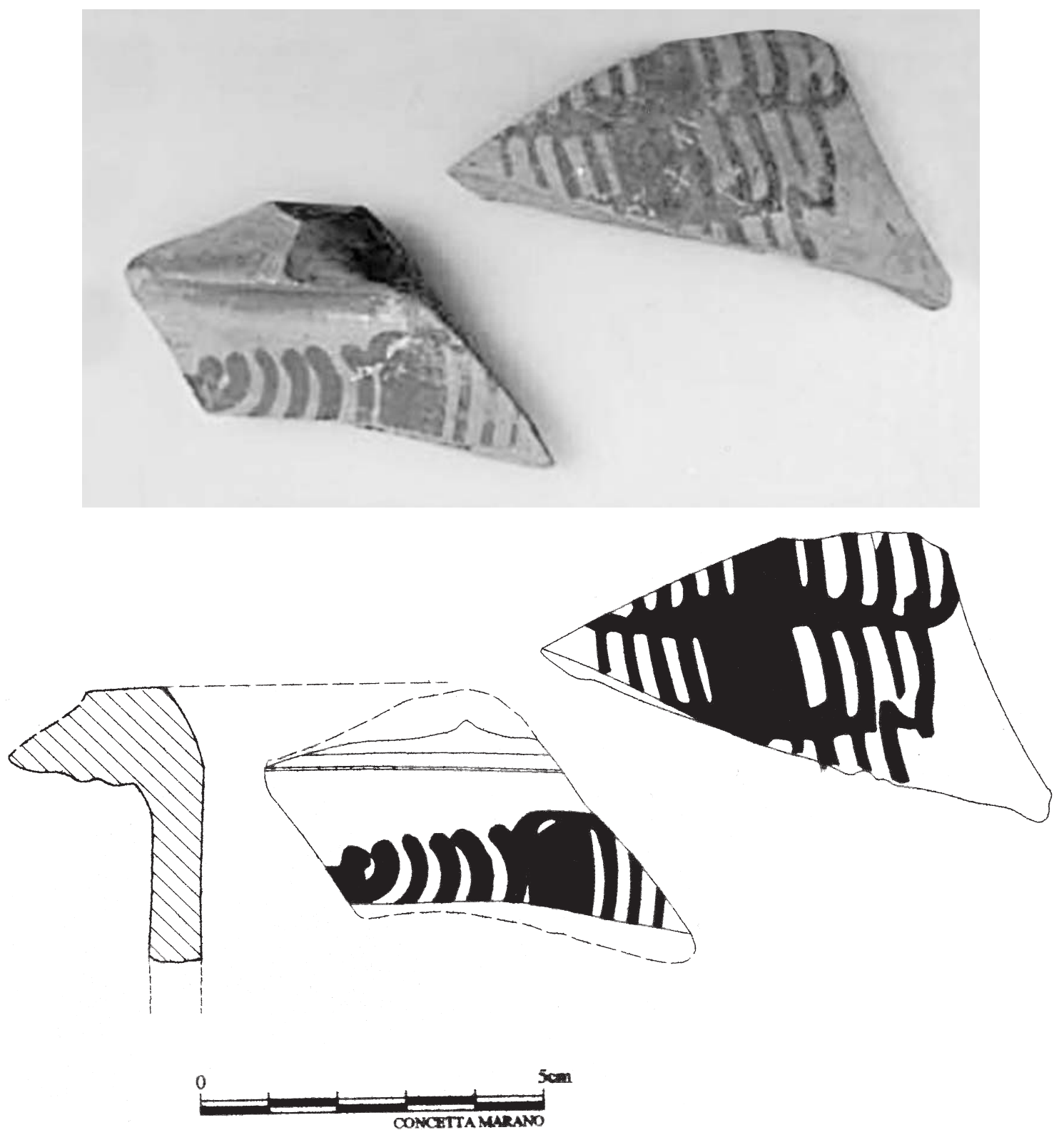

Figura 2 y 3 . Frammenti del kalathos $\mathrm{n}^{\circ} 1$ (?).

Nove frammenti combacianti permettono di ricostruire per circa tre quarti la parte superiore di un kalathos ${ }^{17}$ (fig. 1) con orlo estroflesso, appiattito superiormente e modanato inferiormente, che si restringe verso l'alto ed è decorato, nella parte

${ }^{17}$ Dimensioni generali della porzione di kalathos ricostruita: spess. parete $\mathrm{cm}$. 0.5 ; spess. orlo da $\mathrm{cm} .1 .7 \mathrm{a} \mathrm{cm}$. 0.6; largh. orlo cm. 3; alt. massima conservata cm. 9.7. superiore, da "denti di lupo" piuttosto irregolari. Le anse sono bifide e aderenti alle pareti. La decorazione sulle pareti è realizzata con un pennello multiplo, con una linea centrale più spessa e cinque linee di uguale spessore per lato, alternando gruppi di linee ondulate che, seppure parzialmente, ornano anche le anse, e gruppi di semicerchi penduli. A questo stesso kalathos possono essere attribuiti, per la somiglianza del colore dell'argilla, della vernice e della decorazione, un frammento di parete con gruppo di semicerchi penduli e 

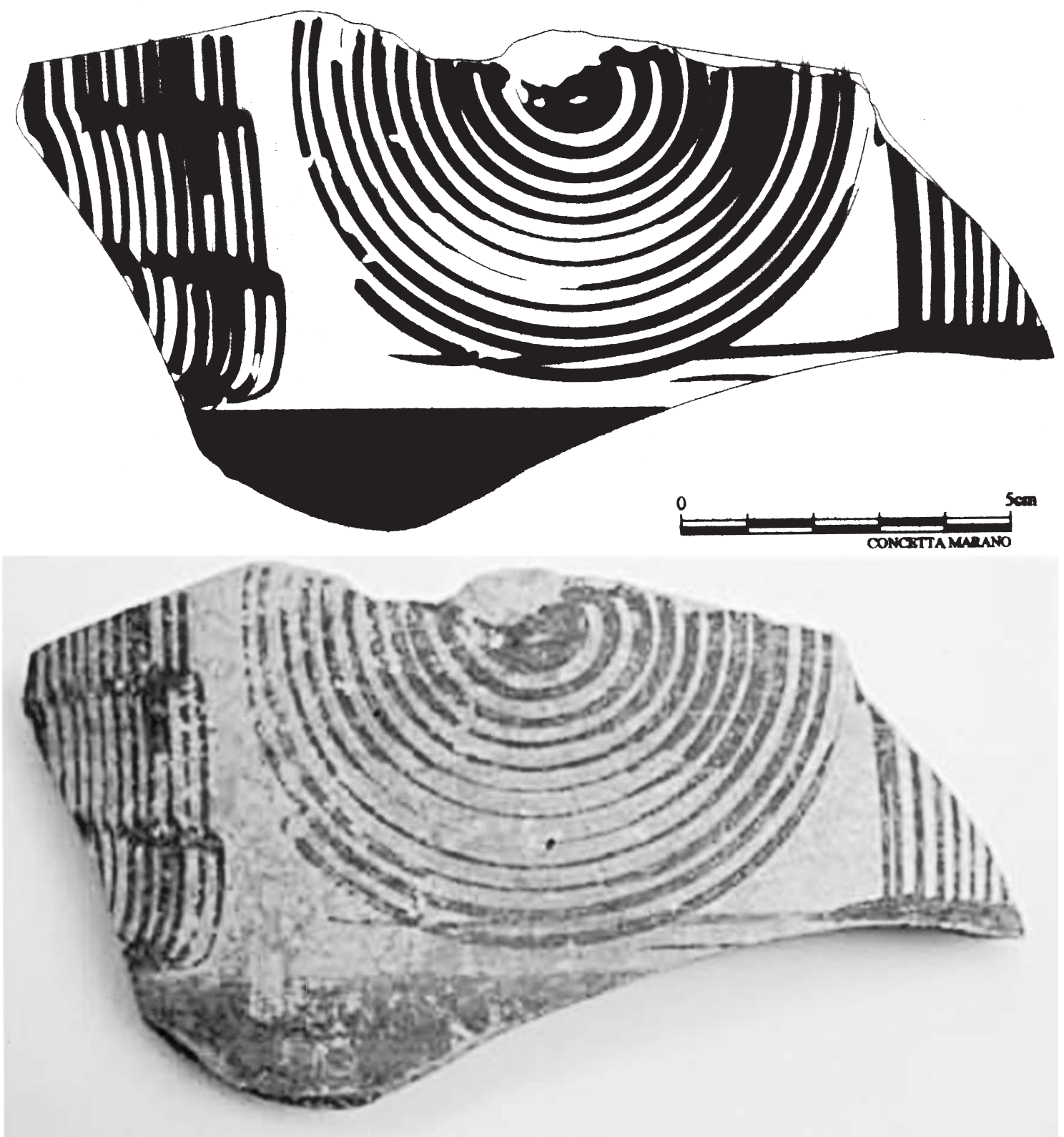

Figura 4. Frammento del kalathos $\mathrm{n}^{\circ} 2$.

con attacco d'orlo (fig. 2), ${ }^{18}$ e un frammento di parete con gruppi di linee ondulate (fig. 3). ${ }^{19}$

${ }^{18}$ Lungh. massima cm. 6.5; alt. massima cm. 4; spess. parete $\mathrm{cm} .0 .6$; alt. orlo all' attacco $\mathrm{cm}$. 1.2; largh. conservata dell'orlo cm. 2.3 .

${ }^{19}$ Lungh. massima cm. 7.4; alt. massima cm. 4.5; spess. parete $\mathrm{cm} .0 .5$.
Al kalathos $\mathrm{n}^{\circ} 2$ (fig. 4) appartiene un frammento di parete ${ }^{20}$ con semicerchi multipli inquadrati da due gruppi di linee ondulate. Il gruppo di semicerchi multipli è composto da undici linee di uguale spessore, con un punto centrale ben evidente. Dato lo stato di conservazione del

${ }^{20}$ Lungh. massima cm. 15.8; alt. massima cm. 7.5; spess. parete $\mathrm{cm} .0 .7$. 


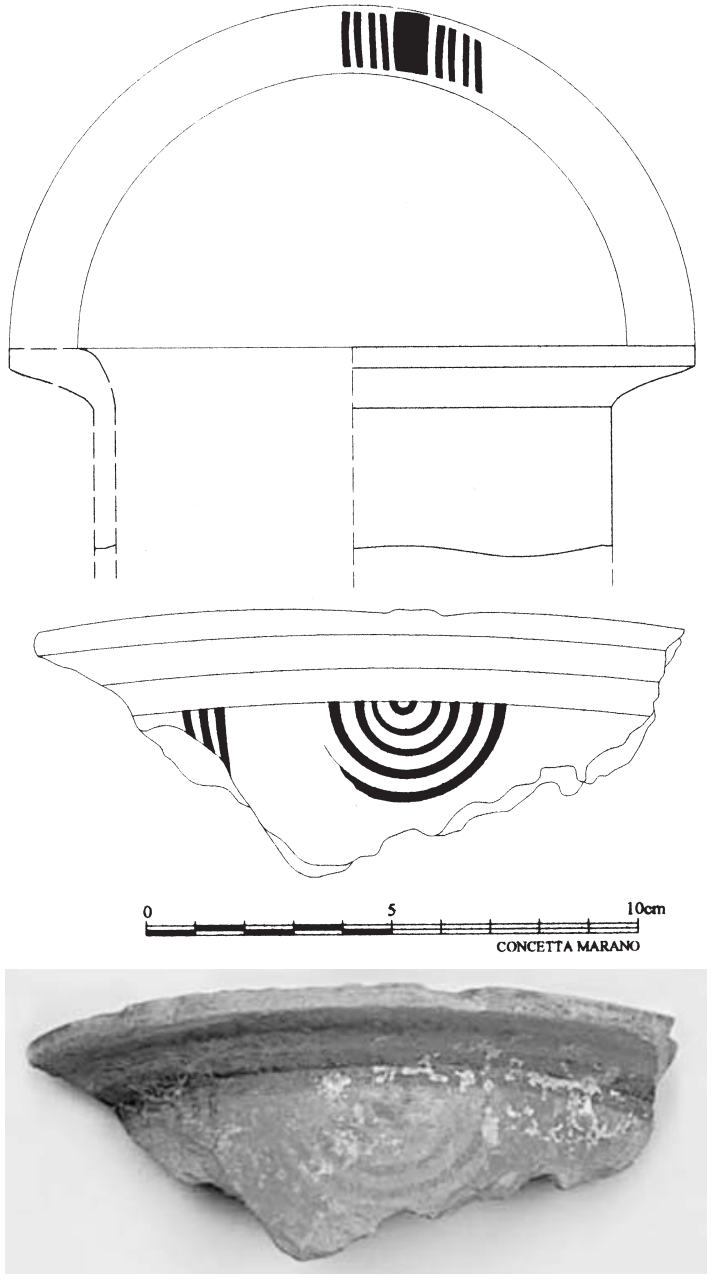

Figura 5. Frammento del kalathos $\mathrm{n}^{\circ} 3$.

frammento, non è possibile invece indicare con sicurezza il numero di linee nei due gruppi: se ne sono conservate undici nel gruppo a sinistra, otto in quello a destra.

Il frammento ${ }^{21}$ del kalathos $\mathrm{n}^{\circ} 3$ (fig. 5) appartiene ad un esemplare di dimensioni inferiori rispetto agli altri due, ma con profilo simile al $n^{\circ} 1$. La decorazione, piuttosto sbiadita, è formata, sull'orlo, da un gruppo di nove linee parallele (con linea centrale di spessore maggiore) e, sulla parete, da almeno cinque semicerchi penduli di eguale spessore e da almeno tre linee di eguale spessore. Un frammento (fig. 6), ${ }^{22}$ per il colore grigiastro ${ }^{23}$ dell' argilla,

${ }^{21}$ Lungh. massima cm. 13; alt. massima cm. 5; spess. parete cm. 0.5, spess. orlo da cm. 1.4 a cm. 0.5; largh. orlo $\mathrm{cm} \mathrm{2.2.}$

${ }^{22}$ Lungh. massima cm. 4.8; alt. massima cm. 4; spess. parete $\mathrm{cm}$. 0.5.

${ }^{23}$ Confrontabile con M. 10R 6/1. sembra non appartenere ai tre esemplari sopra descritti, e potrebbe essere l'unico resto di un quarto kalathos. La decorazione è costituita da un gruppo di linee ondulate dello stesso spessore (se ne conservano sei).

Gli esemplari di Taormina, appartenenti ad un tipo variamente denominato ${ }^{24}$ prodotto in Catalogna soprattutto nel secondo e terzo quarto del II sec. a.C. ${ }^{25}$ si inseriscono perfettamente nel quadro tracciato dalla letteratura scientifica per la distribuzione dei kalathoi iberici. ${ }^{26}$ Come già evidenziato da N. Lamboglia, la presenza della ceramica iberica in Italia «è soprattutto un prodotto della "romanizzazione", che valorizzò anche in Spagna l'economia e le risorse indigene e consentì loro di affermarsi e di espandersi più lontano». ${ }^{27}$ In seguito a questo processo, la ceramica iberica, che ha alle spalle una lunga tradizione,

${ }^{24}$ Tipo 13 (Cuadrado, 1972, 130, 168, tav. 10; Cuadrado Díaz, 1987, 67, fig. 9; Cuadrado Díaz, Quesada Sanz, 1989, 58-59, 87, fig. 14); Forma funzionale 6, forma geometrica 4 (Nordström, 1973, 178-180); Forma IV (Pericot, 1979, 7879); Forma 17b (Aranegui Gasco, Pla Ballester, 1981, 77-79); Gruppo Formale 8. Tipo 8-D (Pereira Sieso, 1988, 160-162, fig. 11, nn. 13-14); Forma-tipo I a, c; II a, b (Ros Sala, 1989, 20-37, 45-48, 73-87); Gruppo A 1-2 (Conde Berdós, 1991, 142-150, in part. 145-147; Ead., 1992, 118-122: l'esemplare $\mathrm{n}^{\circ} 1$, per la presenza delle anse, si inserisce nel Gruppo A-2); Tipo 7. Subtipo 7.1 (Mata Parreño, Bonet Rosado, 1992, 119$122,129-130,153)$.

-I motivi decorativi presenti sui frammenti di kalathoi di Taormina rientrano nelle seguenti classificazioni:

-gruppi di linee ondulate dello stesso spessore: motivo 17 (CVH Liria, 56); tema 8.11 (Nordström, 1973, 242, fig. 32); -gruppi di linee ondulate con linea centrale più spessa: motivo 19 (CVH Liria, 56);

-gruppi di semicerchi con linee multiple dello stesso spessore: motivo 92 (CVH Liria, 104);

-gruppi di semicerchi con linee multiple e linea centrale più spessa: motivo 93 (CVH Liria, 104); tema 5.5 (Nordström, 1973, 239, fig. 29);

"denti di lupo" sull'orlo: motivo 26 (CVH Liria, 56).

I gruppi di linee ondulate (CVA Barcelone 2, tav. 22.2), i gruppi di semicerchi con linee multiple e linea centrale più spessa (CVA Barcelone 2, tav. 22.1-3; tav. 23.1-2; tav. 24.15; tav. 25.1, 4-5, 7; tav. 26.2, 4-5) e le anse bifide o pseudobifide (CVA Barcelone 2, tav. 22.2, tav. 23.1) sono particolarmente frequenti nella produzione dell'atelier di Fontscaldes.

${ }^{25}$ Conde Berdós, 1991, 147; Ead., 1992, 119-120; Ead., 1996, 118.

${ }^{26}$ Sulle caratteristiche e sulla diffusione della ceramica iberica nel II-I sec. a.C., cfr., in particolare: García y Bellido, 1952, 1954, 1957; Lamboglia, 1954; Tarradel, Sanmarti, 1980; Santos Velasco, 1982-1983; Bencivenga Trillmich, 1984, 1985; Conde Berdós, 1989-1990, 1991, 1992, 1996, 1998; Bruni, Conde, 1991.

${ }^{27}$ Lamboglia, 1954, 125. 

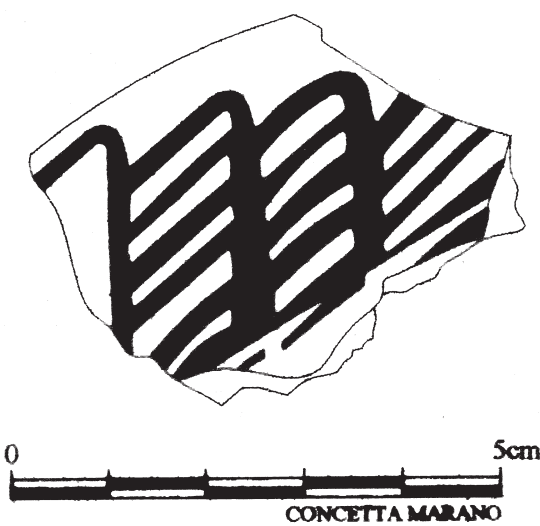

Figura 6. Frammento del kalathos $n^{\circ} 4$ (?).

comincia ad essere esportata soprattutto nel Mediterraneo occidentale, dove è ben attestata, nel II e I sec. a.C., specialmente in siti costieri. La maggiore diffusione della ceramica iberica comporta anche una certa "standardizzazione" della produzione, per cui, tra le numerose forme precedentemente attestate, quella maggiormente prodotta ed esportata è il kalathos ${ }^{28}$, «vaso tipico e quasi "nazionale" degli Iberi in corso di romanizzazione», ${ }^{29}$ mentre la ricca decorazione, anche figurata, della più antica tradizione iberica, cede il passo a motivi geometrici variamente combinati. ${ }^{30}$ Nonostante vari tentativi di considerare il kalathos come contenitore di un non meglio precisabile prodotto iberico, sembra preferibile ipotizzare che questa forma

${ }^{28}$ La predominanza del kalathos tra le forme prodotte ed esportate, sottolineata da N. Lamboglia, 1954, 108, è ribadita, tra gli altri, da Montanya Maluquer, Puig Ochoa, 1979, 223; Santos Velasco, 1982-1983, 147-148; Conde Berdós, 1992, 137; Ead., 1996, 118, 163.

${ }^{29}$ Lamboglia, 1954, 109. Il legame tra la romanizzazione e l'inizio di una più massiccia esportazione della ceramica iberica, già stabilito da N. Lamboglia, è stato comunemente accettato (cfr., ad. es., Conde Berdós, 1991, 163-165; Ead., 1992, 120, 122, 136-138; Ead., 1996, 162). Santos Velasco, 1982-1983, 147 distingue tra il periodo di influenza cartaginese (IV-II sec. a.C.), durante il quale la ceramica iberica ha una circolazione extrapeninsulare molto limitata, e il periodo della romanizzazione (II-I sec. a.C.), che segna l'inizio della "standardizzazione" e della commercializzazione extrapeninsulare della ceramica iberica, in particolare del kalathos.

${ }^{30}$ Cfr., tra gli altri, Montanya Maluquer, Puig Ochoa, 1979, 222-223; Santos Velasco, 1982-1983, 148; Conde Berdós, 1991, 165; Ead., 1998, 318. sia stata esportata in quanto tale, forse anche per il suo carattere "esotico", e non tanto per il suo contenuto. ${ }^{31}$ Dalle carte di distribuzione, per quanto incomplete, ${ }^{32}$ appare chiaro che i kalathoi non sono stati oggetto di un commercio "specializzato" ed esclusivo, ma hanno

${ }^{31}$ Vari studiosi hanno avanzato ipotesi sull'eventuale contenuto dei kalathoi esportati. Ad es., Mezquiriz Irujo, 1955, 13, afferma che i frammenti di Lipari «no pueden aportar ningún dato seguro para la cronología de la expansión de nuestra cerámica en Italia, aunque si pueden ser interesantes como unos testigos más de esta expansión, ya sea exportada como tal cerámica, o más bien como envase de algún producto español apreciado en los mercados italianos»; Del Chiaro, 1973, 65, n. 4 ipotizza che i kalathoi servissero all'esportazione di miele; Montanya Maluquer, Puig Ochoa, 1979, 223 considerano i kalathoi come «envases para una industria local ampuritana (probablemente salazones)»; Tarradell, Sanmarti, 1980, 315, pensano ad un contenuto non meglio precisabile, ma probabilmente non liquido, data la forma espansa del kalathos (frutta secca? miele?); Santos Velasco, 1982-1983, 148, pur non escludendo che i kalathoi siano stati esportati per il loro contenuto (miele, porpora), ipotizza anche un «cierto aprecio como pieza de importación »; Bencivenga Trillmich, 1984, 31 (= Ead., 1985, 554), condivide l'ipotesi: «che ... i 'sombreros de copa' venissero esportati per il loro contenuto - miele o frutta secca»; Mata Parreño, Bonet Rosado, 1992, 129, dopo aver passato in rassegna le varie ipotesi sul contenuto, concludono equilibratamente che «lo más probable es que se trata de un recipiente multifuncional». Altri studiosi, in part. Conde Berdós, 1991, 166; Ead., 1996, 164, sostengono invece che i kalathoi non abbiano viaggiato come contenitore di uno specifico prodotto.

${ }^{32}$ L'incompletezza dei dati pubblicati, e quindi delle carte di distribuzione relative alla ceramica iberica, è più volte evidenziata (ad es. da Bencivenga Trillmich, 1984, 25; Ead., 1985, 552). 
viaggiato insieme ad altri prodotti, forse come merce caricata, nei loro viaggi di ritorno dalla Penisola Iberica, dai commercianti italici, liguri e massalioti. ${ }^{33}$ Non è da escludere, inoltre, che un certo numero di esemplari si sia diffuso accompagnando gli spostamenti di singoli individui, sia per uso personale, sia per piccoli scambi commerciali. ${ }^{34}$ La ceramica iberica è diffusa soprattutto nel Mediterraneo Occidentale, con un forte addensamento in area tirrenica e in centri costieri. Quest'ultima tendenza, unita al numero quasi sempre limitato di esemplari trovato in ciascun sito, può essere considerata una riprova della scarsa penetrazione commerciale di questa ceramica al di fuori della Penisola Iberica. ${ }^{35}$

In Sicilia, i kalathoi iberici sono solo pochi esemplari anche in siti estesamente scavati e ben pubblicati. Già a proposito dei kalathoi di Tindari, M.A. Mezquiriz osserva che si tratta di un prodotto «de uso esporádico en la ciudad de Tyndaris», come dimostra il fatto che tali frammenti sono solo una minima percentuale, in rapporto alle altre classi ceramiche, e che essi sono stati trovati all'interno della stessa abitazione. Anche a Lipari e ad Heraclea i kalathoi iberici sono solo pochi esemplari, se paragonati alla notevole quantità di materiale edito. A Taormina, all'interno di una cisterna che ha restituito migliaia di reperti ceramici, i kalathoi iberici sono appena tre o quattro.

Tutti i kalathoi rinvenuti in Sicilia provengono, secondo una tendenza ben attestata, da centri costieri. Con le dovute cautele, si può notare inoltre la concentrazione dei rinvenimenti in centri della Sicilia settentrionale, e ciò potrebbe essere riferito al già evidenziato addensamento della ceramica iberica nell'area tirrenica del Mediterraneo. La presenza di frammenti di kalathoi ad Heraclea Minoa sarebbe invece da mettere in relazione, secondo $\mathrm{C}$. Bencivenga Trillmich, con l'esistenza di una rotta nord-africana per la diffusione della ceramica iberica, ben attestata a Cartagine $^{36} \mathrm{e}$ in altri centri. ${ }^{37}$

In assenza di ulteriori dati e di uno studio globale su tutte le altre produzioni iberiche eventualmente presenti nell'isola, è meglio evitare di avanzare

\footnotetext{
${ }^{33}$ Lamboglia, 1954, 125. Ipotesi condivisa, tra gli altri, da Bencivenga Trillmich, 1984, 30-31; Ead., 1985, 553.

${ }^{34}$ Conde Berdós, 1991, 166; Ead., 1992, 138; Ead., 1996, 164.

35 Santos Velasco, 1982-1983, 147; Bencivenga Trillmich, 1984, 30; Ead., 1985, 553; Conde Berdós, 1996, 162.

${ }^{36}$ Bencivenga Trillmich, 1984, 31, n. 31 (= Ead., 1985, 553); Morel 1990, p. 24, tav. 2e.

${ }^{37}$ Ad es. a Lixus, cfr. Aranegui Gascó (ed.) 2005, pp. 97106
}

interpretazioni più specifiche sui kalathoi iberici in Sicilia, ${ }^{38}$ limitandosi a considerare tale presenza come un fenomeno normale in un contesto di scambi mediterranei.

\section{BIBLIOGRAFIA ${ }^{39}$}

ARANEGUI GASCO, C., PLA BALlESTER, E., 1981: La cerámica ibérica. In "La baja época de la cultura ibérica". Actas de la mesa redonda celebrada en conmemoración del décimo aniversario de la Asociación Española de amigos de la arqueología. (Madrid 1979). Madrid.

ARANEGUI GASCÓ, C., (ed..), 2005: Lixus-2 Ladera Sur. Excavaciones arqueológicas marroco-españolas en la colonia fenicia. Campañas 2000-2003. Valencia. (Saguntum Extra 6).

BENCIVENGA TRILLMICH, C., 1984: La ceramica iberica da Velia. Contributo allo studio della diffusione della ceramica iberica in Italia. $M M, 25,20-33$.

BENCIVENGA TRILLMICH, C., 1985: Observaciones sobre la difusión de la cerámica ibérica en Italia. XVII Congreso Nacional de Arqueología. (Logroño 1983), Zaragoza, 551-556.

BRUNI, S., CONDE, M. J., 1991: Presencia ibérica en Etruria y el mundo itálico a través de los hallazgos cerámicos de los siglos III-I a.C. In "La presencia de material etrusco en la Penísula Ibérica" (José Remesal, Olimpio Musso eds.). Barcelona.

CONDE BERDÓS, M. J., 1989-1990: La producció ceràmica ibèrica tardana (segles II-I a.C.) a les comarques de Tarragona. Acta arqueologica de Tarragona, 3, 33-39.

CONDE BERDÓS, M. J., 1991: Les produccions de kálathoi d'Empúries i la seva difusió mediterrània (segles II-I a.C.). Cypsela, 9, 141-168.

CONDE BERDÓS, M. J., 1992: Una producció ceràmica característica del món ibèric tardà: el kalathos «barret de copa». Fonaments, 8, 117-170.

CONDE BERDÓS, M. J., 1996: La cerámica ibérica de Albintimilium y le tráfico mediterráneo en los siglos II-I a.C. RStLig, 62, 115-168.

\footnotetext{
${ }^{38}$ Mezquiriz, 1953, 159, ad es., ipotizza che la ceramica iberica a Tindari vada messa in relazione con la presenza di mercenari iberici in Sicilia. García y Bellido, 1954, 251, per rinforzare questa ipotesi, cita i soldati di Sertorio che, dopo la sconfitta ad opera di Pompeo, sono sbarcati in Sicilia e sono stati perseguitati da Verre (Cic. Verr. 5, 72, 146 e 154). Tale ipotesi, perfettamente legittima, è solo una delle spiegazioni possibili.

${ }^{39}$ Per le abbreviazioni delle riviste si fa riferimento alla Archäologische Bibliographie.
} 
CONDE BERDÓS, M. J., 1998: Estado actual de la investigación sobre la cerámica ibérica pintada de época plena y tardía. Revista de Estudios Ibéricos, 3, 299-335.

CUADRADO DÍAZ, E., 1987: La necrópolis ibérica de «El Cigarralejo» (Mula, Murcia). Madrid (Biblioteca Præhistorica Hispana 23).

CUADRADO DÍAZ, E., QUESADA SANZ, F., 1989: La cerámica ibérica fina de "El Cigarralejo" (Murcia). Estudio de cronología. Verdolay, Revista del museo de Murcia, 1, 49-115.

CUADRADO, E., 1972: Tipologia de la cerámica ibérica fina de "El Cigarralejo". Mula (Murcia). TrabPrehist, 29, 125-187.

CVA Barcelone 2, 1958-1965: de C. Serra i Ràfols, J.; Colominas i Roca, J., CVA Espagne. Musée Archéologique de Barcelone 2. Barcelone.

CVH Liria, 1954: Ballestrer Tormo, I.; Fletcher Valls, D.; Pla Ballestrer, E.; Jorda Cerda, F.; Alcacer Grau, J., Corpus Vasorum Hispanorum. Ceramica del Cerro de San Miguel. Liria. Madrid.

DE MIRO, E., 1958: Heraclea Minoa. - Scavi eseguiti negli anni 1955-56-57. NSc, s. VIII, 12, 232-287.

DEL CHIARO, M. A., 1973: An Iberian Sherd in Yougoslavia. AJA, 77, 65-66.

DI STEFANO, C. A., 1976: Marsala. Ricerche Archeologiche al Capo Boeo. SicA, 32, 25-31.

GARCÍA Y BELLIDO, A., 1952: Nuevos datos sobre la cronología final de la cerámica ibérica y sobre su expansión extrapeninsular. ArchEspA, 25, 39-45.

GARCÍA Y BELLIDO, A., 1954: Expansión de la cerámica ibérica por la cuenca occidental del Mediterráneo. ArchEspA, 27, 246-254.

GARCÍA Y BELLIDO, A., 1957: Estado actual del problema referente a la expansión de la cerámica ibérica por la cuenca occidental del Mediterráneo. ArchEspA, 30, 90-106.

LAMBOGLIA, N., 1954: La ceramica iberica negli strati di Albintimilium e nel territorio ligure e tirrenico. RStLig, 20, 83-125.

MATA PARREÑO, C., BONET ROSADO, H., 1992: La cerámica ibérica: ensayo de tipología. In "Estudios de Arqueología ibérica y romana. Homenaje a Enrique Pla Ballester". Valencia, 117173 (Serie de trabajos varios 89).
Meligunìs Lipára IX.1, 1998: Bernabò-Brea, L., Cavalier, M., Villard, F., Meligunìs Lipára. Vol. IX. Topografia di Lipari in età greca e romana. Parte I L'Acropoli. Palermo.

Meligunìs Lipára IX.2, 1998: Bernabò-Brea, L., Cavalier, M., Meligunìs Lipára. Vol. IX. Topografia di Lipari in età greca e romana. Parte II - La città bassa. Palermo.

MEZQUIRIZ IRUJO, M. A., 1955: Cerámica ibérica en Lípari. ArchEspA, 28, 112-113.

MEZQUIRIZ, M. A., 1953: Cerámica ibérica en Tyndaris (Sicilia). ArchEspA, 26, 156-161.

MONTANYA MALUQUER, R., PUIG OCHOA, M.R., 1979: La cerámica ibérica pintada tardía y sus perduraciones. RStLig, 45, 221-230.

MOREL, J.-P., 1990: L'apport des fouilles de Carthage à la chronologie des céramiques héllenistiques. B' E $\pi \sum u v E \lambda \lambda K \varepsilon \rho$ (Rhodos 1989), Athina, pp. 1730.

NORDSTRÖM, S., 1973: La céramique peinte ibérique de la province d'Alicante. II. Stockholm, Almqvist \& Wiksell (Acta Universitatis Stockholmiensis. Stockholm Studies in Classical Archaeology 8).

PEREIRA SIESO, J., 1988: La cerámica ibérica de la cuenca del Guadalquivir. I. Propuesta de clasificación. TrabPrehist, 45, 143-173.

PERICOT, L., 1979: Cerámica ibérica. Barcelona.

ROS SALA, M. M., 1989: La pervivencia del elemento indígena: la cerámica ibérica. Murcia (La ciudad romana de Carthago Nova: fuentes y materiales para su estudio 1).

SANTOS VELASCO, J. A., 1982-1983: La difusión de la cerámica ibèrica pintada en el Mediterráneo Occidental. CuadCastellon 9, 135-148.

TARRADEL, M., SANMARTI, E., 1980: L'état actuel des études sur la céramique ibérique. In "Céramiques Hellénistiques et Romaines" (Centre de Recherches d'Histoire Ancienne 36 - Annales Littéraires de l'Université de Besançon 242), 303330 .

Recibido el 8-05-06 Aceptado el 13-06-06 